\title{
Recurrent DNA copy number changes revealed by comparative genomic hybridization in primary Merkel cell carcinomas
}

\author{
Marcelo L Larramendy ${ }^{1,2, *}$, Virve Koljonen ${ }^{4, *}$, Tom Böhling ${ }^{3}$, Erkki Tukiainen ${ }^{4}$ and \\ Sakari Knuutila ${ }^{1}$ \\ ${ }^{1}$ Department of Medical Genetics, Haartman Institute, University of Helsinki and Helsinki University Central \\ Hospital Laboratory Diagnostics, Helsinki, Finland; ${ }^{2}$ Laboratorio de Citogenética y Cátedra de Citología, \\ Facultad de Ciencias Naturales y Museo, Universidad Nacional de La Plata, La Plata, Argentina; \\ ${ }^{3}$ Department of Pathology, Haartman Institute and Helsinki University Central Hospital, University of \\ Helsinki, Helsinki, Finland and ${ }^{4}$ Department of Plastic Surgery, Helsinki University Hospital Helsinki, \\ Finland
}

\begin{abstract}
Comparative genomic hybridization (CGH) was used to search for gains, high-level amplifications and losses of DNA sequences along all chromosome arms in 19 primary Merkel cell carcinomas (MCC). Extensive genetic aberrations, with a mean value of $5.5 \pm 1.1$ changes per tumor were detected in 13 out of the 19 samples analyzed. Our CGH results reveal several new and other previously known chromosomal regions that are involved in the pathogenesis of MCC. The majority of the alterations were gains of whole chromosomes or whole chromosome arms. Compared to losses, the frequency of DNA copy number gains was two-fold. DNA sequence copy number gains were most common in chromosomes $6(42 \%), 1(37 \%)$, and $5(32 \%)$. The most frequent minimal common regions of gains were 6 pterqter $(42 \%), 1 q 11 q 31(32 \%)$, and $5 p(32 \%)$. No recurrent high-level amplifications were observed. High-level amplifications of small chromosomal regions were found in four samples out of the 19 tumors analyzed (21\%). Amplifications affected 1q22q24 (5\%), 4p (5\%), and 5p (5\%). Losses most frequently affected chromosomes $13(21 \%)$ and $4(16 \%)$. Minimal common regions with the most frequent losses were 13q13q31 (21\%), $4 q(16 \%)$, and 16q $(11 \%)$. No significant statistical correlation between genomic aberrations and clinicopathological factors was revealed, despite the fact that there was an obvious tendency towards it. Primary MCC expressing DNA alterations were predominantly distinguished in large tumors, and risk of metastatic dissemination was three-fold compared to tumors with no DNA alterations. Modern Pathology (2004) 17, 561-567, advance online publication, 5 March 2004; doi:10.1038/modpathol.3800091
\end{abstract}

Keywords: Merkel cell carcinoma; comparative genomic hybridization; prognosis

Merkel cell carcinoma (MCC) is a rare, aggressive primary neuroendocrine tumor of the skin. Its annual incidence varies between 0.2 and 0.45 per 100000 people. $^{1,2}$ Its natural course, particularly in large tumors, is rapid progression of primary tumor and metastasis to regional lymph nodes. ${ }^{3,4}$ This tumor is usually located in the sun-exposed areas of the body; half of the tumors are found in the head and neck region. ${ }^{4-6}$ The elderly are most commonly

Correspondence: S Knuutila, Department of Medical Genetics, University of Helsinki, PO Box 21 (Haartmaninkatu 3, 4th floor), Helsinki FIN-00014, Finland.

E-mail: sakari.knuutila@helsinki.fi

*Equal contribution.

Received 9 October 2003; revised and accepted 31 December 2003; published online 5 March 2004 affected by this disease. ${ }^{7}$ Mean age varies from 70 to 80 years in recent studies. ${ }^{4,8}$

The prognosis is rather poor. Depending on the length of the follow-up period, up to $40-50 \%$ of the patients develop a local recurrence, $55 \%$ regional lymph node metastasis and $36 \%$ distant metastasis. ${ }^{6}$ Overall survival is associated with the stage of disease at presentation and with sex, but not with age. ${ }^{9,10}$

Large tumor size $(>2 \mathrm{~cm}$ ) has been found to have a detrimental effect on survival in MCC patients. ${ }^{11,12}$ Radical surgery is the recommended procedure to improve prediction of better survival in MCC. ${ }^{13,14}$

Previously, cytogenetic analyses have not shown any recurrent reciprocal translocations in MCC that would generate fusion genes or lead to activation of oncogenes. Little is thus known about the chromosomal and genetic alterations in MCC. 
The aim of the present study was to perform a CGH analysis to obtain a genome-wide pattern of chromosomal gains, losses and high-level amplifications of small chromosomal areas in a cohort of 19 primary MCC. A further aim was to find out whether these chromosomal aberrations correlate with patient outcome and whether they could serve as prognostic markers.

\section{Materials and methods}

\section{Tumor Specimens and Patient Characteristics}

The material consisted of 19 primary MCC samples obtained from 19 Finnish patients treated at the Departments of Plastic Surgery, Helsinki University Central Hospital and Vaasa Central Hospital between 1987 and 2002, with complete clinical data. Treatments, operations, and development of recurrences and/or metastasis were documented.

None of the patients had received chemo- or radiotherapy before operation. The histological diagnosis was confirmed by two researchers (TB and VK) by using hematoxylin-eosin (HE) ${ }^{15}$ stained slides. In addition, immunohistochemical verification was done for all the samples using cytokeratin20 (CK-20) antibody. Immunohistochemical staining for thyroid transcription factor-1 (TTF-1) was used to exclude small-cell lung carcinoma (SCLC) metastasis. ${ }^{16}$ This was negative in all cases. Size was measured from HE slides and it was recorded as $<2$ or $\geq 2 \mathrm{~cm}$.

In HE staining, the typical histology representation of the MCC samples was small blue cells with sparse cytoplasm, dispersed chromatin, monotonous, medium-sized, round-to-oval nuclei and abundant mitoses in the dermis with frequent extension to subcutaneous tissue.

Clinical characteristics of the patients are presented in Table 1.

The mean follow-up was 2.7 years (range 7 days to 10 years). There were 10 (53\%) male and nine (47\%) female patients. The median age of the patients was 68 years (range 59-97 years). Size of the primary tumor varied from 0.8 to $6.5 \mathrm{~cm}$, mean tumor size $2.5 \mathrm{~cm}$. Eight $(42 \%)$ of the tumors were located in the head and neck region. Eight tumors were located in the extremities (five in the upper and three in the lower extremities) and three (15\%) were in the trunk. Metastatic dissemination was noted in two of the patients at the presentation. Seven more patients developed metastatic course of disease, altogether $47 \%$ progressed to metastasis. Local recurrence was recorded in eight $(42 \%)$ patients.

The cut-off value of $2 \mathrm{~cm}$ (also the median) was chosen, for small and large tumor groups as our earlier clinicopathological study showed statistical correlation between tumors $\geq 2 \mathrm{~cm}$ in size and unfortunate course of disease. ${ }^{12}$

DNA were extracted from paraffin-embedded tissue sections following the procedure reported by Isola et l. $^{17}$

\section{Comparative Genomic Hybridization}

Comparative genomic hybridization (CGH) was performed using direct fluorochrome-conjugated DNA for all samples according to the protocol described elsewhere with minor modifications. ${ }^{18}$ Briefly, tumor DNA and reference DNA (genomic DNA from peripheral blood leukocytes from normal donors) were labelled by nick translation with

Table 1 Clinical characteristics of the 19 MCC patients

\begin{tabular}{|c|c|c|c|c|c|c|c|}
\hline $\begin{array}{l}\text { Sample } \\
\text { number }\end{array}$ & Age (years) & Sex & $\begin{array}{l}\text { Size of the } \\
\text { primary tumor }(\mathrm{cm})\end{array}$ & $\begin{array}{l}\text { Location of the } \\
\text { primary tumor }\end{array}$ & Recurrence & Metastasis & Follow-up (months) \\
\hline 1 & 78 & $\mathrm{M}$ & 1 & Left eyebrow & & & 18 \\
\hline 2 & 77 & $\mathrm{M}$ & 1.4 & Left antebrachium & & & 8 \\
\hline 3 & 81 & $\mathrm{~F}$ & 0.8 & Right buttock & & & 68 \\
\hline 4 & 68 & $\mathrm{M}$ & 1 & Right wrist & & & 19 \\
\hline 5 & 67 & $\mathrm{M}$ & 1.5 & Left cheek & & Yes & 10 \\
\hline 6 & 80 & $\mathrm{~F}$ & 1.5 & Left cheek & Yes & Yes & 56 \\
\hline 7 & 64 & $\mathrm{~F}$ & 1 & Right eye & Yes & & 126 \\
\hline 8 & $78^{\mathrm{a}}$ & $\mathrm{F}$ & 2 & Right breast & & Yes & 0.25 \\
\hline 9 & 59 & $\mathrm{M}$ & 4 & Right breast & & & 25 \\
\hline 10 & 79 & $\mathrm{M}$ & 4 & Left brachium & Yes & Yes & 7 \\
\hline 11 & 69 & $\mathrm{M}$ & 2 & Left antebrachium & Yes & Yes & 14 \\
\hline 12 & 85 & $\mathrm{M}$ & 6.5 & Nose & Yes & Yes & 12 \\
\hline 13 & 74 & $\mathrm{~F}$ & 5.5 & Left eyebrow & & Yes & 14 \\
\hline 14 & 97 & $\mathrm{~F}$ & 3 & Right knee & Yes & & 14 \\
\hline 15 & 86 & $\mathrm{~F}$ & 3 & Right cheek & & & 16 \\
\hline 16 & $81^{\mathrm{a}}$ & $\mathrm{F}$ & 6 & Left thigh & Yes & Yes & 13 \\
\hline 17 & 79 & M & 2 & Right thigh & & Yes & 11 \\
\hline 18 & 82 & M & 1.2 & Right antebrachium & Yes & & 65 \\
\hline 19 & 70 & $\mathrm{~F}$ & 1 & Right ear & & & 132 \\
\hline
\end{tabular}

M: male; F: female.

${ }^{\mathrm{a}}$ Metastatic dissemination at the presentation. 
fluorescein-iso-thiocyanate (FITC)-conjugated dCTP and dUTP (DuPont, Boston, MA, USA), and Texas Red-conjugated dCTP and dUTP (Dupont), respectively, to obtain fragments ranging from 600 to $2000 \mathrm{bp}$ as previously reported. ${ }^{19}$ The hybridization mixture consisted of $400 \mathrm{ng}$ of tumor DNA, $400 \mathrm{ng}$ of reference DNA, and $10 \mu \mathrm{g}$ of unlabelled human Cot-1 DNA (Gibco/BRL, Life Technologies, Gaithersburg, MD, USA) dissolved in $10 \mu \mathrm{l}$ of hybridization buffer ( $50 \%$ formamide, $10 \%$ dextran sulphate, $2 \times$ SSC). The hybridization mixture was denatured at $75^{\circ} \mathrm{C}$ for $5 \mathrm{~min}$ and hybridized to a slide with normal metaphase spreads denatured in $70 \%$ formamide/ $2 \times \mathrm{SSC}(\mathrm{pH} 7)$ at $68^{\circ} \mathrm{C}$ for $2 \mathrm{~min}$. Hybridization was carried out at $37^{\circ} \mathrm{C}$ for $48 \mathrm{~h}$. The slides were then washed three times in $50 \%$ formamide/ $2 \times \mathrm{SSC}$ $(\mathrm{pH} 7)$, twice in $2 \times \mathrm{SSC}$, and once in $0.1 \times \mathrm{SSC}$ at $45^{\circ} \mathrm{C}$, followed by $2 \times \mathrm{SSC}, 0.1 \mathrm{M} \mathrm{NaH} \mathrm{PO}_{4}-0.1 \mathrm{M}$ $\mathrm{Na}_{2} \mathrm{HPO}_{4}-0.1 \%$ Nonidet P-40 (pH 8) and distilled water at room temperature for $10 \mathrm{~min}$ each. After airdrying, the slides were counterstained with $4^{\prime}, 6-$ diamidino-2-phenyl-indole-dihydrochloride (DAPI) (Sigma Chemical Co., St Louis, MO, USA), and mounted with an antifading medium (Vectashield ${ }^{\mathbb{R}}$, Vector Laboratories, Burlingame, CA, USA).

\section{Digital Image Analysis}

The hybridization was analyzed using an Olympus fluorescence microscope and the ISIS digital image analysis system (MetaSystems $\mathrm{GmbH}$, Altlussheim, Germany), based on an integrated high-sensitivity monochrome charge-coupled device (CCD) camera and automated CGH analysis software. Three-color images (green for tumor DNA, red for reference DNA, and blue for counterstaining) were acquired from 12 metaphases per sample. The chromosomal regions were interpreted as over-represented when the green-to-red ratio exceeded 1.17 (gains) or 1.5 (high-level amplifications) and as under-represented (losses) when the ratio was less than 0.85 . In each CGH experiment, a negative (peripheral blood DNA from normal donor) control and a positive (tumor DNA with known copy number changes) control were included and run simultaneously with the tumor samples. Telomeric and heterochromatinic regions were excluded from the analysis when they appeared as the sole aberration present in the sample as these regions cannot be evaluated reliably by $\mathrm{CGH}{ }^{20,21}$ DNA copy number changes involving $16 \mathrm{p}$ and 19 were excluded from the analysis unless they represented a high-level amplification. ${ }^{22,23}$ All results were confirmed using a 99\% confidence interval with $1 \%$ error probability. Briefly, intraexperimental standard deviations for all positions in the CGH ratio profiles were calculated from the variation of the ratio values of all homologous chromosomes within the experiment. Confidence intervals for the ratio profiles were then computed by combining them with an empirical interexperi- mental standard deviation and by estimating the error probability based on the $t$-distribution.

\section{Statistical Analysis}

The association between tumors with no DNA changes, tumors with changes in chromosomes 6 , $1,5,13$, and 4 and with clinical outcome was tested by Fisher's exact test. End point for the surveillance was chosen for the detection of recurrences and/or metastasis. Statistical analyses were performed using the NCSS 2000 software program (NCSS Statistical Software, Kaysville, UT, USA). The chosen level of significance was $P<0.05$, unless otherwise noted.

\section{Results}

\section{Overview of DNA Sequence Copy Number Changes}

Of the 19 samples, 13 (68.4\%) had changes with a mean value of $5.5 \pm 1.1$ aberrations per sample (range 1-12). Six samples (31.6\%) did not show any aberrations. Compared to losses, the frequency of DNA copy number gains was two-fold (gains:losses =1.8:1). All chromosomal regions with increased or decreased DNA sequence copy number changes are summarized in Figure 1. Table 2 shows the copy number karyotypes and Table 3 the most frequent chromosomal regions of gains, high-level amplifications, and losses.

The group of small tumors $(<2 \mathrm{~cm})$ comprised nine samples (Nos. 1-7, 18, and 19) and the remaining 10 samples (Nos. 8-17) were large tumors. Of the six samples that showed no DNA changes, only one was a large tumor, the other five

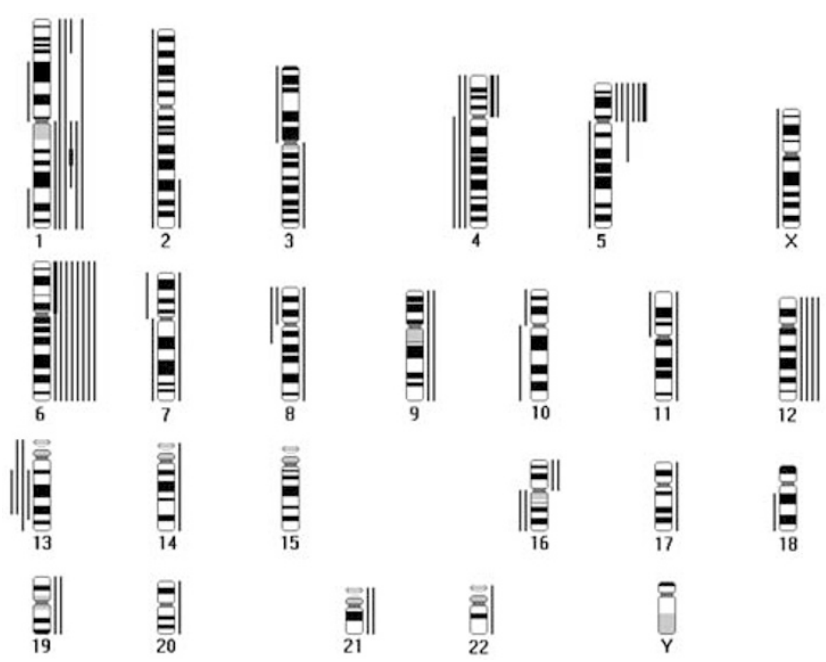

Figure 1 Summary of the gains and losses of DNA sequence copy number in 19 MCC samples analyzed by CGH. Losses are shown on the left and gains on the right of each involved chromosome. Each line represents a genetic aberration seen in one sample. High-level amplifications are shown in thick lines. 
Table 2 DNA sequence copy number changes in 19 primary MCC samples analyzed by CGH

\begin{tabular}{ll}
\hline Sample number & DNA copy number changes, CGH results \\
\hline 1 & No changes \\
2 & No changes \\
3 & revish enh(1q,5p),dim(7q,13q13q32) \\
4 & No changes \\
5 & revish enh(1,5p,17,21),dim(5q,7q,10p,13),amp(4p) \\
6 & dim(X) \\
7 & No changes \\
8 & revish enh(2q32qter,5pterq15,6),dim(4,16q),amp(6p) \\
9 & revish enh(1,3q,5p,6,8),dim(2,3p,4,7p,11p,13pterq31) \\
10 & rev ish enh(6,12,19,21) \\
11 & revish enh(1pterp33,1q11q31,16p)dim(1p31p11,1q32qter,4q,16q),amp(1q22q24) \\
12 & revish enh(1q,6,9,11,12,16p),dim(8p,10q) \\
13 & revish enh(6) \\
14 & rev ish enh(5p,6,12) \\
15 & rev ish enh(5p,6),amp(5p) \\
16 & rev ish enh(1,4p,6,7,9,12,14,19,20,22),dim(13q13q31,18q12-qter) \\
17 & No changes \\
18 & No changes \\
19 & rev ish dim(8pterq13) \\
\end{tabular}

F: female; M: male.

Table 3 Most frequent gains, high-level amplifications, and losses of DNA sequence copy number detected by CGH in 19 primary MCC samples

\begin{tabular}{|c|c|c|c|c|c|}
\hline \multicolumn{2}{|c|}{ Gains (\%) } & \multicolumn{2}{|c|}{ High-level amplifications (\%) } & \multicolumn{2}{|c|}{ Losses (\%) } \\
\hline Location $^{\mathrm{a}}$ & Number of samples & Location $^{\mathrm{a}}$ & Number of samples & Location $^{\mathrm{a}}$ & Number of samples \\
\hline 6 & $8(42)$ & $1 q 22 q 24$ & $1(5)$ & $13 q 13 q 31$ & $4(21)$ \\
\hline 1q11q31 & $6(32)$ & $4 p$ & $1(5)$ & $4 q$ & $3(16)$ \\
\hline $5 p$ & $6(32)$ & $5 p$ & $1(5)$ & $4 p$ & $2(11)$ \\
\hline 1q32qter & $5(26)$ & $6 p$ & $1(5)$ & $8 \mathrm{p}$ & $2(11)$ \\
\hline 1pterp33 & $4(21)$ & & & $16 q$ & $2(11)$ \\
\hline 12 & $4(21)$ & & & & \\
\hline $4 p$ & $2(11)$ & & & & \\
\hline 9 & $2(11)$ & & & & \\
\hline $16 p$ & 2 (11) & & & & \\
\hline $19 \mathrm{p}$ & $2(11)$ & & & & \\
\hline 21 & $2(11)$ & & & & \\
\hline
\end{tabular}

${ }^{\mathrm{a}}$ Locations indicate minimal common regions.

were small tumors, revealing that $55 \%$ of the small tumors showed no DNA changes. Of those tumors that showed no genomic changes only $17 \%$ progressed to metastatic disease.

No significant statistical correlation was distinguished between the CGH aberrations and clinical features $(P>0.05)$. The relative risk (RR) for developing metastatic dissemination in the presence of DNA alterations was 3.0.

\section{Gains and High-level Amplifications of DNA}

Gains of DNA sequence copy number were most commonly observed in chromosomes $6(42 \%), 1$ (37\%), $5(32 \%)$, and $12(21 \%)$. The most frequent minimal common regions were 6pterqter $(42 \%)$, $1 q 11 q 31(32 \%)$, $5 p(32 \%)$, and 1q32qter $(26 \%)$.
Other minimal common regions of gains are presented in Table 3.

All DNA sequence copy number gains in chromosome 6 were seen in the large tumors. Five patients $(63 \%)$ with gains in chromosome 6 developed metastatic course of disease and four (66\%) with gains in chromosome 1 advanced to metastatic dissemination. Relations between DNA changes and recurrence and metastasis are presented in Figure 2. High-level amplifications of small chromosomal regions were found in four of the 19 tumors analyzed (21\%) (Table 3 ).

\section{Losses of DNA}

Losses most frequently affected chromosomes 13 $(21 \%)$ and $4(16 \%)$. Minimal common regions with the most frequent losses were 13q13q31 (21\%), 4q 


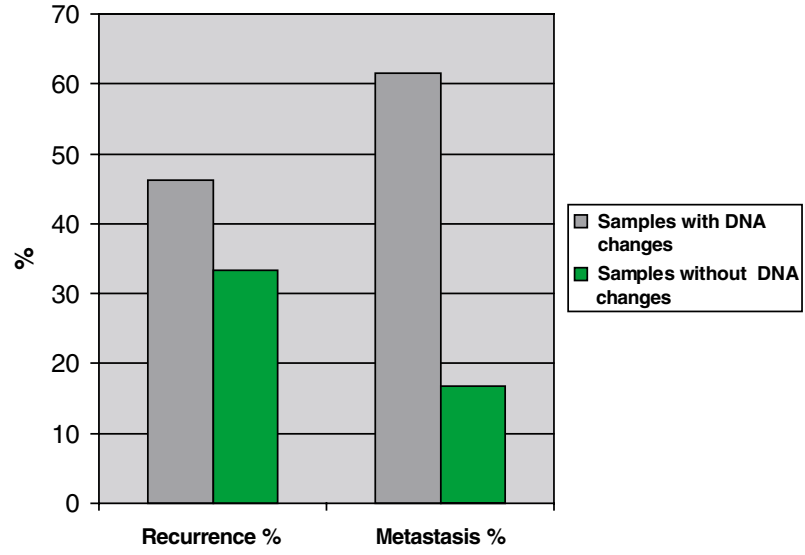

Figure 2 Relations between DNA changes and clinical features, recurrences, and metastasis.

(16\%), and $16 q(11 \%)$ (Table 2). All the losses affecting chromosome 4 were in the large tumor group. Patients with losses in chromosomes 13 and 4 , respectively, 50 and $66 \%$, progressed to metastasis.

\section{Discussion}

Our study reports the first CGH analysis performed on a large series of primary MCC. The analysis revealed that 13 of the $19(68 \%)$ MCC samples showed gains and/or losses of DNA sequences involving at least one, but typically several different chromosome regions. On average, the samples had five copy number aberrations per sample, with almost all chromosome arms involved. Most of the genomic abnormalities $(9 / 13,69 \%)$ were found in large tumors (size $\geq 2 \mathrm{~cm}$ ).

In addition to previously known chromosomal regions, our $\mathrm{CGH}$ results revealed several new recurrent DNA copy number sequences that may be involved in the pathogenesis of MCC.

Gains of DNA sequences affected mainly whole chromosomes or chromosome arms, whereas highlevel amplifications and losses were rare. The present study reveals a characteristic pattern of genomic imbalances, including over-representation of chromosomes 6, 1q, 5p, 1p, 12, 4p, 9, 16p, 19p, and 21, in order of frequency. Frequent loss of DNA sequences was observed in $13 q, 4 q$, and $16 q$, in order of frequency. High-level amplifications were rare, affecting $4 p, 5 p$, and $6 p$ or narrowed down to $1 \mathrm{q} 22 \mathrm{q} 24$.

The clinical characteristics of our patients reported previously, ${ }^{12}$ are well consistent with other studies. Slight male predominance, mean age 68 years and almost half of the tumors located in the head and neck region. ${ }^{11,14}$ During the course of the disease $47 \%$ of the patients progressed to metastatic dissemination, local recurrence was recorded in
$42 \%$ patients. These data are also consistent with previous studies. ${ }^{4,24}$

Of the six tumors that showed no genomic aberrations, five $(83 \%)$ were found in the small tumors group. Five patients (55\%) with small tumors (size $<2 \mathrm{~cm}$ ) showed no DNA changes. In compliance with previous studies, ${ }^{11,25}$ large tumor size and nodal disease at the presentation had a negative impact on overall survival in MCC patients.

A three-fold risk was observed between genetic alterations and poor clinical outcome. Although we failed to demonstrate a significant statistical correlation between genomic aberrations and unfortunate course of disease, probably due to small number of specimens, there was an obvious tendency towards it.

Trisomy of chromosome 6 is widely considered as recurrent chromosomal aberration in MCC, ${ }^{26-28}$ which was the case also in our study. In $42 \%$ of the primary MCC samples analyzed by CGH, overrepresentation of all genomic material of chromosome 6 was observed. Our study showed that aberration in chromosome 6 was distinguished solely in large tumors (size $\geq 2 \mathrm{~cm}$ ). Five $(63 \%)$ of those patients developed metastasis in the course of disease.

The present analysis showed that half of the eight tumors with an increased copy number affecting the whole chromosome 6 , had a gain of the whole chromosome 12, suggesting that the presence of simultaneous gains of chromosomes 6 and 12 may contribute to the development and progression of MCC.

Six of the tumor samples showed at least one gain of DNA sequence copy number affecting either the whole chromosome 1 or different chromosomal regions in $1 \mathrm{p}$ and 1q. Although trisomy 1 appears to be characteristic of MCC, its specific role is still unknown. It is thought that it confers proliferative advantages on the tumor cells in MCC. ${ }^{29}$ In our material, $66 \%$ of the patients with gains in chromosome 1 progressed to nodal disease.

Gains of $5 p$ material found in our present study have also been reported by others. ${ }^{30,31}$ Another previously observed recurrent abnormality is the entire or partial loss of 13q. In our samples, the most frequent losses of DNA sequence copy number were located at 13q13q31, which agree well with the results by others. ${ }^{30,32,33}$

We observed recurrent losses, albeit infrequently, affecting chromosome 4 (with the minimal common region $4 \mathrm{q}$ ) and in chromosome 8 (minimal common region $8 \mathrm{p}$ ). So far, no tumor suppressor gene has been identified on chromosome 4. Additionally, each loss in chromosome 4 was identified in the large tumor group. Two-thirds of those patients developed metastasis.

MCC and SCLC are both neuroendocrine carcinomas that share some similarities in their clinical behavior. Deletions of chromosome $3 p$ are detected in almost every SCLC $^{34,35}$ and $3 p$ allele loss is 
considered universal in the pathogenesis of all lung carcinoma types. ${ }^{36}$

In our material only one patient, patient No. 9 (Table 2), presented with deletion of chromosome $3 p$. This finding is contrary to a previous study that reported $\mathrm{LOH}$ on $3 p$ to be a common abnormality in primary MCC. ${ }^{37}$ It seems that primary MCC does not share the deletion on $3 p$ with other similar neuroendocrine carcinomas, that is, SCLC. It has been postulated that chromosome $3 p$ harbors a critical tumor suppressor gene involved in the pathogenesis of human lung cancers. ${ }^{38}$ The pathogenesis of MCC seems to have different route from other neuroendocrine carcinomas.

In conclusion, it could be suggested that the activation of genes leading to the initiation and progression of MCCs is mostly related with the presence of extra copies of several chromosomes or chromosomal arms rather than with amplifications of small chromosomal areas. Finally, the possibility of losses of tumor-suppressor genes cannot be ruled out because of deletions beyond the resolution of the CGH method or their locations at telomeric regions, both conditions not detectable by CGH.

\section{Acknowledgements}

This work was supported by the Helsinki University Central Hospital, Finnish Cancer Society, Sigrid Jusélius Foundation and K.A. Johansson Foundation, in Finland, and by the National Council of Scientific and Technological Research (CONICET) and the National University of La Plata (Grant number 11/N325) in Argentina. We thank Mr Timo Pessi for assistance with the statistical analysis.

\section{References}

1 Pan D, Naryan D, Ariyan S. Merkel cell carcinoma: five case reports using sentinel lymph node biopsy and review of 110 new cases. Plastic Reconstr Surg 2002;110:1259-1265.

2 Chuang TY, Su WP, Muller SA. Incidence of cutaneous $\mathrm{T}$ cell lymphoma and other rare skin cancers in a defined population. J Am Acad Dermatol 1990;23 (2 Part 1):254-256

3 Smith DE, Bielamowicz S, Kagan R, et al. Cutaneous neuroendocrine (Merkel cell) carcinoma. A report of 35 cases. Am J Clin Oncol 1995;18:199-201.

4 Akhtar S, Oza KK, Wright J. Merkel cell carcinoma: report of 10 cases and review of the literature. J Am Acad Dermatol 2000;43(5 Part 1):755-767.

5 Eftekhari F, Wallace S, Silva EG, et al. Merkel cell carcinoma of the skin: imaging and clinical features in 93 cases. Br J Radiol 1996;69:226-233.

6 Gillenwater AM, Hessel AC, Morrison WH, et al. Merkel cell carcinoma of the head and neck: effect of surgical excision and radiation on recurrence and survival. Arch Otolaryngol Head Neck Surg 2001; 127:149-154.
7 Carpentier O, Carrotte-Lefebvre I, Patenotre P, et al. Primitive cutaneous neuroendocrine carcinomas or Merkel's tumor. Clinical and therapeutic aspects in 22 patients. Presse Med 2002;31:735-739.

8 Hohaus K, Kostler E, Schonlebe J, et al. Merkel cell carcinoma-a retrospective analysis of 17 cases. J Eur Acad Dermatol Venereol 2003;17:20-24.

9 Hitchcock CL, Bland KI, Laney III RG, et al. Neuroendocrine (Merkel cell) carcinoma of the skin. Its natural history, diagnosis, and treatment. Ann Surg 1988;207:201-207.

10 Medina-Franco H, Urist MM, Fiveash J, et al. Multimodality treatment of Merkel cell carcinoma: case series and literature review of 1024 cases. Ann Surg Oncol 2001;8:204-208.

11 Tai PT, Yu E, Tonita J, et al. Merkel cell carcinoma of the skin. J Cutan Med Surg 2000;4:186-195.

12 Koljonen V, Böhling T, Granhroth G, et al. Merkel cell carcinoma: a clinicopathological study of 34 patients. Eur J Surg Oncol 2003;29:607-610.

13 Tai PTH, Yu E, Tonita J, et al. Merkel cell carcinoma of the skin. J Cutan Med Surg 2000;4:186-195.

14 Brissett AE, Olsen KD, Kasperbauer JL, et al. Merkel cell carcinoma of the head and neck: a retrospective case series. Head Neck 2002;24:982-988.

15 He L, Tuckett RP, English KB. Chemosensitivity of the rat type I slowly adapting mechanoreceptor. Biol Signals Receptors 1999;8:382-389.

16 Cheuk W, Kwan MY, Suster S, et al. Immunostaining for thyroid transcription factor 1 and cytokeratin 20 aids the distinction of small cell carcinoma from Merkel cell carcinoma, but not pulmonary from extrapulmonary small cell carcinomas. Arch Pathol Lab Med 2001;125:228-231.

17 Isola J, DeVries S, Chu L, et al. Analysis of changes in DNA sequence copy number by comparative genomic hybridization in archival paraffin-embedded tumor samples. Am J Pathol 1994;145:1301-1308.

18 Larramendy ML, Tarkkanen M, Valle J, et al. Gains, losses, and amplifications of DNA sequences evaluated by comparative genomic hybridization in chondrosarcomas. Am J Pathol 1997;150:685-691.

19 Larramendy ML, Huhta $\mathrm{T}$, Vettenranta $\mathrm{K}$, et al. Comparative genomic hybridization in childhood acute lymphoblastic leukemia. Leukemia 1998;12: 1638-1644.

20 Larramendy ML, El-Rifai W, Knuutila S. Comparison of fluorescein isothiocyanate- and Texas redconjugated nucleotides for direct labeling in comparative genomic hybridization. Cytometry 1998;31: 174-179.

21 Kallioniemi A, Kallioniemi O-P, Piper J, et al. Detection and mapping of amplified DNA sequences in breast cancer by comparative genomic hybridization. Proc Nat Acad Sci USA 1994;91:2156-2160.

22 Kallioniemi O-P, Kallioniemi A, Piper J, et al. Optimizing comparative genomic hybridization for analysis of DNA sequence copy number changes in solid tumors. Genes Chromosomes Cancer 1994;10: 231-243.

23 Kallioniemi A, Kallioniemi O-P, Citro G, et al. Identification of gains and losses of DNA sequences in primary bladder cancer by comparative genomic hybridization. Genes Chromosomes Cancer 1995;12: 213-219.

24 Helmbold P, Schroter S, Holzhausen HJ, et al. Merkel cell carcinoma. Hautarzt 2002;53:652-658. 
25 Ott MJ, Tanabe KK, Gadd MA, et al. Multimodality management of Merkel cell carcinoma. Arch Surg 1999;134:388-392 (discussion 392-393).

26 Moll I, Bohnert E, Herbst C, et al. Establishment and characterization of two Merkel cell tumor cultures. J Invest Dermatol 1994;102:346-353.

27 Gancberg D, Feoli F, Hamels J, et al. Trisomy 6 in Merkel cell carcinoma: a recurrent chromosomal aberration. Histopathology 2000;37:445-451.

28 Harle M, Arens N, Moll I, et al. Comparative genomic hybridization (CGH) discloses chromosomal and subchromosomal copy number changes in Merkel cell carcinomas. J Cutan Pathol 1996;23:391-397.

29 Schlegelberger B, Bartels H, Sterry W. Chromosomal evolution in a Merkel cell carcinoma. Cancer Genet Cytogenet 1994;75:74-76.

30 Van Gele M, Speleman F, Vandesompele J, et al. Characteristic pattern of chromosomal gains and losses in Merkel cell carcinoma detected by comparative genomic hybridization. Cancer Res 1998;58: 1503-1508.

31 Popp S, Waltering S, Herbst C, et al. UV-B-type mutations and chromosomal imbalances indicate common pathways for the development of Merkel and skin squamous cell carcinomas. Int J Cancer 2002;99:352-360.

32 Van Gele M, Leonard JH, Van Roy N, et al. Combined karytyping, CGH and M-FISH analysis allows detailed characterization of unidnetified chromosomal rearrangements in Merkel cell carcinoma. Int J Cancer 2002;101:137-145.

33 Leonard JH, Leonard P, Kearsley JH. Chromosomes 1, 11 , and 13 are frequently involved in karyotypic abnormalities in metastatic Merkel cell carcinoma. Cancer Genet Cytogenet 1993;67:65-70.

34 Onuki N, Wistuba II, Travis WD, et al. Genetic changes in the spectrum of neuroendocrine lung tumors. Cancer 1999;85:600-607.

35 Wiest JS, Franklin WA, Drabkin H, et al. Genetic markers for early detection of lung cancer and outcome measures for response to chemoprevention. J Cell Biochem Suppl 1997;28-29:64-73.

36 Wistuba II, Behrens C, Virmani AK, et al. High resolution chromosome $3 p$ allelotyping of human lung cancer and preneoplastic/preinvasive bronchial epithelium reveals multiple, discontinuous sites of $3 \mathrm{p}$ allele loss and three regions of frequent breakpoints. Cancer Res 2000;60:1949-1960.

37 Leonard JH, Williams G, Walters MK, et al. Deletion mapping of the short arm of chromosome 3 in Merkel cell carcinoma. Genes Chromosomes Cancer 1996;15: 102-107.

38 Oh JJ, West AR, Fishbein MC, et al. A candidate tumor suppressor gene, H37, from the human lung cancer tumor suppressor locus 3p21.3. Cancer Res 2002;62: 3207-3213. 